\title{
Impacts of interpersonal distancing on-board trains during the COVID-19 emergency: case studies and viewpoints from industry, consultancies and public transportation companies in Italy
}

Pierluigi Coppola ( $\sim$ pierluigi.coppola@polimi.it)

Polytechnic of Milan https://orcid.org/0000-0003-0729-282X

Francesco De Fabiis

Polytechnic of Milan

\section{Research Article}

Keywords: COVID-19, interpersonal distancing, High-speed Rail, suburban rail services, demand forecast, train capacity

Posted Date: September 29th, 2020

DOI: https://doi.org/10.21203/rs.3.rs-84680/v1

License: (c) (i) This work is licensed under a Creative Commons Attribution 4.0 International License.

Read Full License 


\section{Abstract}

The emergency from COVID-19 and the cities lockdown have had a strong impact on transportation sector. Travel demand, in particular, registered an unprecedented overall contraction, especially in passenger transportation which has dramatically dropped down with peaks of less $90 \%-95 \%$. In the reopening phase, travel demand is gradually resuming the levels before the crisis, but some structural changes are observed both in travel behavior and in transportation supply. This paper aims at giving a contribution to the undergoing debate by presenting the results of a study to forecast the impacts of keeping an interpersonal distancing of one-meter between passengers on board (sub-urban and highspeed) trains. The results of the study have been shared and discussed with a panel of chief executives of industries, consultancies in the mobility sector and Public Transportation (PT) companies. It has emerged that interpersonal distancing and the reduced level of capacity on board vehicles are seen as effective to prevent the contagion, but are perceived as not sustainable due to the induced increase in operating costs. This is confirmed by the simulation carried out during the study which have showed the need of new urban policies not only limited to the transportation domain (e.g. changes in the times of schools, commerce and industries) in order to manage the demand peaks at the stations and on-board vehicles.

\section{Introduction}

In the recent months, following the outbreak of COVID-19 emergency, a number of measures have been implemented to contain the spread of the virus: in the peak phases of contagion, commerce, schools and other activities were closed (i.e. "cities lockdown") and non-essential travels were banned, except in case of urgent need. In the re-opening phase, businesses and activities partially re-open and travel demand gradually resumes, but some structural changes in both transportation supply and travel behavior are observed. In China, for instance, the fear of contagion has shifted travelers from public transportation to private vehicles with an increase of car usage from $34 \%$ to $66 \%$ (Ipsos, 2020). Similar trends can be observed in Italy, where the first evidence showed a negative perception in terms of safety in the use of public transportation, especially with respect to traveling by car or on foot (Isfort, 2020): in fact, on a scale of 1 to 10 , traveling by bus, metro or train is perceived as very unsafe (between 3.1 and 3.5 on average), while the opposite occurs for journeys with private cars and on foot (8.7 and 7.3 as average ratings) (Table 1 ).

Table 1 - Travel safety perception, by modes of transportation (Likert scale: from 1=very unsafe to $10=$ very safe) - (Source Isfort: 2020 - adapted) 


\begin{tabular}{cc}
\hline Safety perception index & Average \\
\hline car & 8,7 \\
on foot & 7,3 \\
bus/tram & 3,5 \\
metro & 3,1 \\
train (long distance) & 3,3 \\
\hline
\end{tabular}

Specific mitigation measure to prevent contagion from COVID-19 have been introduced to maintain interpersonal distancing on-board public transportation vehicles, in particular on trains and metro. In Italy, this distance was set at one meter, thus significantly reducing vehicle capacity between $50 \%$ and $20 \%$ of the pre-crisis level (Figure 1) and heavily impacting on the public transportation supply, both at urban, regional and national scale.

This paper aims at giving a contribution to the undergoing debate by presenting the results of a study on the impacts of interpersonal distancing and the viewpoint of a selected number of stakeholders about the possible measures to be implemented in the re-opening phase.

The paper is organized as follows. In section 2 the background and the research methodology of the study are described, including the results of a survey carried out between April and May 2020 involving a panel of chief executive officers (CEOs) of industries, consultancies in the mobility sector, and Public Transportation (PT) companies in Italy. Section 3 presents the results of the study on the impacts forecasting analysis of interpersonal distancing on two railways lines (suburban and high-speed rail), including the stakeholder's views about its effectiveness and sustainability. In Section 4 results are discussed and some conclusions are drawn, providing ideas for future research directions.

\section{Background}

The motivation for a study on the impacts of interpersonal distancing on-board sub-urban and highspeed trains came out of a survey consisting of a focus group and an on-line questionnaire (Coppola and De Fabiis, 2020).

The focus group consisted of an open discussion moderated by an expert, set up around what are the factors that most influenced supply (i.e. products and services) and demand (i.e. customers and travelers) following the COVID-19 emergency, and what are the changes on the whole transportation system due to the health emergency and what contribution could come from different business (i.e. industry, consultancy, Public Transportation companies).

A very negative common sentiment was observed with reference to the short term. However, different views emerged in the medium period (April 2021) between industry/consultancies and Public Transportation (PT) companies: the formers showed more optimism towards the future thanks to the opportunities for developing new products after the crisis. As regards industries, the most promising businesses are those generated by an expected increase in demand for both means of transportation 
suitable for urban micro-mobility (for example, scooters) and private and public electric vehicles (i.e. cars and buses).

On the other hand, there is great uncertainty about the future according to PT companies, worried about losses in terms of passengers and consequently of ticketing revenues. The estimated loss of passenger demand is between $40 \%$ and $50 \%$ for regional services, while on long-distance services it is between $75 \%$ and $85 \%$. The panel opinion was that demand the levels are far from returning equal to those before the crisis, even in the long term.

The attitudes and expectations emerged from the focus group, were further investigated by means of a detailed survey, carried out two weeks after the focus group, aimed at probing:

- the expected impacts on transportation sector businesses in terms of supply (products /services) and demand (customers /travelers) and, in general, the criticalities that can be envisaged in the reopening phases on the overall transportation system;

- the effectiveness and economic sustainability of some measures proposed for dealing with the emergency, such as:

- differentiated fares by time-of-day;

- seat reservation or reservation of travel-time slots to access stations;

- use of protection mask and scan of passengers' body temperature;

- interpersonal distancing on-board the vehicles and sanitization of vehicles;

- demand-responsive services or dedicated home-to-work shuttle services;

○ increasing PT service frequency to improve PT capacity.

To measure the perception of effectiveness and sustainability by the respondents, a Likert scale from 1 to 5 has been adopted, where 1 corresponds to "very low" effectiveness/sustainability, 2 "low", 3 "neutral", 4 "high" and 5 "very high". The result is that, according to the panel's evaluation, the proposed measures can fall within four domains (Figure 2).

It is possible to distinguish between measures with:

- High effectiveness and High sustainability $(\mathrm{HH})$;

- High effectiveness and Low sustainability (HL);

- Low effectiveness and High sustainability (LH);

- Low effectiveness and Low sustainability (LL).

In particular, the $\mathrm{HH}$ domain contains both measures such as the obligation to reserve a seat or to reserve the travel-time slot to access vehicles or stations, and the introduction of fares differentiated by time-ofday. However, some companies within the panel have highlighted problems regarding the correct applicability of these measures, in particular for those that require enforcement outside the station to 
regulate access. The LL domain, low effectiveness and low sustainability, is populated only by the measure regarding the introduction of demand-responsive or dedicated home-to-work services, considered as neither effective nor sustainable by the respondents.

To cope with the limited capacity of public transportation, due to the need to maintain interpersonal distancing, the increase in PT service frequency is judged to be effective but not sustainable in both the short and medium term. This is due to the current limited number of drivers and vehicles, whose hiring / purchasing would require lengthy administrative procedures.

The sanitization of vehicles, the use of protection masks and the scan of passengers' body temperature fall within the LH domain and are seen as unsustainable measures due to both the lack of personnel and the increase in operating costs. Among the proposed measures, keeping interpersonal distancing on board the vehicles is perceived as the worst in terms of sustainability, albeit quite effective, and for this reason, in this paper, the impacts that the introduction of this measure would generate on the PT systems are probed. Note that, in the panel opinion, in order to efficiently and effectively cope with the reduced capacity of the means of transportation, due to the introduction of interpersonal distancing measures, demand peaks should be smoothed either by reducing volumes (e.g. by promoting smart-working) and by distributing the flows along the entire daytime (e.g. by changing the times of the cities).

\section{Investigating The Impacts Of Interpersonal Distancing On-board Trains}

With the aim of investigating under which demand levels and daily distribution, the impact of interpersonal distancing could lead to the over-saturation of Public Transportation systems either onboard vehicles or at the stations, the simulation-based procedure shown in Figure 3 was proposed.

Two case studies with a different seat reservation system have been considered: a suburban railway line where no seat reservation is required, and a High-speed Rail (HSR) line for which seat reservation is mandatory. Different criticalities might be arising in the two cases: in the former (suburban line case) train could get overcrowded and therefore not compliant with the interpersonal distancing provisions; in the second case (HSR line) part of the potential demand might be unsatisfied due to seats unavailability, and, in turn, be shifted to others modes with consequent loss of revenue for the operators.

From the demand side, the PRE-COVID scenario with passenger flows level equal to those prior to the lockdown was taken as the upper bound, while the PHASE 1 scenario was taken as the lower bound with only essential activities re-opened and assuming very low demand level (i.e. those observed in the first days after the lockdown). The intermediate demand scenarios have been built up by considering a gradual relaxation of the restrictions on activities and trips.

\subsection{The case of the sub-urban railway line S13}


The S13 suburban railway line connects Bovisa (Milan) with Pavia passing through the city center of Milan. Figure 4 shows in detail the path of the line, including stops and interconnections with other public transportation services.

The reconstruction of the PRE-COVID and PHASE 1 scenarios was based on historical data relating to boarded and alighted passengers at each station and for each train. Data analyzed for the PRE-COVID scenario were related to November 2019, while data for the PHASE-1 scenario refer to March 2020. A reduction of the maximum number of on-board passengers in a working day of about $79 \%$ is observed from the PRE-COVID scenario to the PHASE-1 scenario, for both directions Pavia-Mi Bovisa and Mi Bovisa - Pavia (Figure 5). However, the structure of the demand did still present the same two peaks periods: a morning peak (from 6:30 to 9:00 am, on the Pavia - Mi Bovisa direction) and an evening peak (from 17:00 to 19:00 on the Mi Bovisa-Pavia direction).

In the simulated scenarios, in addition to the PRE-COVID and PHASE 1 scenarios, the following scenarios have been simulated:

- PARTIAL RE-OPENING, in which it is assumed a partial reopening of university and schools, i.e. half of the students attending in presence and half in distance mode, resulting in a demand level for study purpose of $50 \%$ less than that assumed in the PRE-COVID scenario;

- NO-STUDENTS, in which it is assumed that schools and university would be kept closed and teaching activities go entirely in distance mode, thus resulting in a null demand for study purpose in order to reduce trains loads in particular during the peak periods.

The estimated station-to-station OD matrices in the peak periods have been assigned to the trains using a schedule-based assignment model (Cascetta and Coppola, 2012) in order to estimate the flow on the trains operating in the morning (6:30-9:00) and evening (17:00-19:00) peak periods considered (Figure 6).

Service frequency and rolling stock (i.e. "Treno Servizio Regionale") TSR composed by five coaches 2 with a cab and a wheelchair bay for disable people $(\mathrm{MCH})$ and the others without $(\mathrm{M})$, are considered as invariant. A full train capacity, including all available seats and considering an occupancy rate of 4 passengers per square meter for the standing places, results in 960 passengers/train. The introduction of interpersonal distancing measures yields a train capacity of 270 passengers, assuming half of the seats available plus an aliquot of standees equal to the $10 \%$ of the seats available in normal conditions. Under these restrictions it can be observed a severe overload of the train in almost all the stations and for almost all the trains running in the peak periods.

Table 2 reports the aggregate results for the simulated scenarios, in terms of number of stations with departing trains in overcrowded conditions and of average number of passengers exceeding train capacity: even assuming zero trips for study purposes, there would be a number of stations ( 6 and 5 out of 13, respectively on the Pavia - Mi Bovisa and Mi Bovisa - Pavia direction) with departing trains exceeding the allowed capacity of 270 passengers, with an average number of overcrowding ranging 
between 12 and 47 passengers/train. These prove that the adoption of the interpersonal distancing is not sustainable even considering a complete limitation of students' mobility.

Table 2 - Outputs of the scenario simulations with capacity restrictions (i.e. 270 passenger/train) under different assumption of demand levels.

\section{Pavia - Bovisa (6:30-9:00)}

\begin{tabular}{lcccc}
\hline & \multicolumn{2}{c}{ Demand level } \\
\hline & $\begin{array}{c}\text { PRE- } \\
\text { COVID }\end{array}$ & $\begin{array}{c}\text { PARTIAL } \\
\text { RE- } \\
\text { OPENING }\end{array}$ & $\begin{array}{c}\text { NO- } \\
\text { STUDENTS }\end{array}$ & $\begin{array}{c}\text { PHASE } \\
1\end{array}$ \\
\hline $\begin{array}{l}\text { Number of stations with oversaturated train (\# } \\
\text { stations) }\end{array}$ & 11 & 9 & 6 & 0 \\
\hline $\begin{array}{l}\text { Average number of passengers over train } \\
\text { capacity (pax/train) }\end{array}$ & 157 & 116 & 47 & 0 \\
\hline
\end{tabular}

\section{Bovisa - Pavia (17:00-19:00)}

\section{Demand level}

\begin{tabular}{lcccc}
\hline & $\begin{array}{c}\text { PRE- } \\
\text { COVID }\end{array}$ & $\begin{array}{c}\text { PARTIAL } \\
\text { RE- } \\
\text { OPENING }\end{array}$ & $\begin{array}{c}\text { NO- } \\
\text { STUDENTS }\end{array}$ & $\begin{array}{c}\text { PHASE } \\
1\end{array}$ \\
\hline $\begin{array}{l}\text { Number of stations with oversaturated train (\# } \\
\text { stations) }\end{array}$ & 9 & 7 & 5 & 0 \\
$\begin{array}{l}\text { Average number of passengers over train } \\
\text { capacity (pax/train) }\end{array}$ & 102 & 48 & 12 & 0 \\
\hline
\end{tabular}

\subsection{The case of the HSR Lines: Milan-Rome-Naples}

The Milan-Naples high-speed Rail (HSR) line connects some of the most attractive Italian cities, such as Bologna, Florence and Rome (Cascetta et al., 2013). In this study the direct services between Rome-Milan and Rome-Naples have been considered.

The invariant scenarios have been identified and assessed based on the timetable of HSR services offered by the two HSR undertakings, i.e. the incumbent Trenitalia and NTV (Nuovo Trasporto Viaggiatori): a decrease of about $90 \%$ of services per day was observed from the PRE-COVID to the PHASE 1 scenario (Figure 7).

With reference to the rolling stock, the two undertakings operate the service with different rolling stock: ETR500 and ETR1000 trains for Trenitalia, with a capacity of 597 and 467 seats, respectively; AGV575 and ETR675 trains for NTV, with capacities of 462 and 472 seats, respectively. 
In the simulated scenarios, a capacity reduction of $50 \%$ of the seats available was considered due to the introduction of interpersonal distancing measures. Moreover, HSR service frequency has been assumed equal to 24 trains/day on the Milan-Rome line, and equal to 29 trains/day on the Rome-Naples one.

On the demand side, in addition to the PRE-COVID scenario, two scenarios have been simulated which respectively consider the $50 \%$ and $40 \%$ of the PRE-COVID demand level.

The supply-demand interaction has been simulated by means of a schedule-based run choice model (Cascetta \& Coppola, 2012), allowing to estimating the on-board passengers for each train during the day. Figure 8 shows, for instance, the estimated demand flows in the Scenario $40 \%$, disaggregated by hour-ofday, including those users that could not travel on HSR due to unavailability of seats (i.e. unsatisfied demand).

It can be observed that on the Rome-Milan and Milan-Rome directions there is quite a significative loss of passengers respectively $24 \%$ and $20 \%$ (i.e. unsatisfied demand), whereas on the Rome-Naples line this is limited only to the Naples to Rome direction, with a loss of $17 \%$.

Table 3 shows the unsatisfied demand by section and direction in the simulated scenarios. It can be observed that:

- in all the simulated scenarios, the most affected sections are those connecting Rome and Milan;

- the unsatisfied demand is very significant when the volumes are assumed equal to those prior to the pandemic (PRE-COVID), with a peak of $62 \%$ on the Rome-Milan direction;

- in the reduced demand scenarios, the unsatisfied demand is between $20 \%$ and $30 \%$ on the RomeMilan-Rome line and between $0 \%$ and $20 \%$ on the Rome-Naples-Rome line.

Table 3 - Percentages of passengers lost by HSR undertakings, in the simulated scenarios, for each direction.

\begin{tabular}{lcccc}
\cline { 2 - 5 } Demand levels & $\begin{array}{c}\text { Rome } \\
\text { Milan }\end{array}$ & $\begin{array}{c}\text { Milan }- \\
\text { Rome }\end{array}$ & $\begin{array}{c}\text { Rome - } \\
\text { Naples }\end{array}$ & $\begin{array}{c}\text { Naples - } \\
\text { Rome }\end{array}$ \\
\cline { 2 - 5 } $\begin{array}{l}\text { PRE-COVID SCENARIO } \\
\text { (100\%) }\end{array}$ & $62 \%$ & $49 \%$ & $38 \%$ & $16 \%$ \\
SCENARIO 50\% & $30 \%$ & $25 \%$ & $19 \%$ & $0 \%$ \\
SCENARIO 40\% & $24 \%$ & $20 \%$ & $17 \%$ & $0 \%$ \\
\hline
\end{tabular}

\section{Discussion And Conclusions}

Based on the results of a survey presented among the CEO of industries, consultancies in the mobility sector, and major PT companies operating in the Lombardy Region, this papers focuses on one of the measures that was considered among the most effective to contain the spread of the virus on Public 
Transportation, but, on the other hand, unsustainable from both economic and operational perspectives, i.e. keeping interpersonal distancing on-board public transportation vehicles.

The results of the simulations presented have been carried out for two different case studies: a suburban railway line (urban case) and an HSR line (ex-urban case), differentiated by the characteristic of the demand (urban vs. ex-urban) and by the seat reservation system (i.e. free access vs. mandatory reservation).

In the case of the suburban line, the simulation has shown that the adoption of the interpersonal distancing is not sustainable even with drastic limitations of students' mobility. In fact, even assuming zero trips for study purposes, there would be a number of stations ( 6 and 5 out of 13 , respectively on the Pavia-Mi Bovisa and Mi Bovisa-Pavia direction) with departing trains exceeding the allowed capacity of 270 passengers, with an average number of overcrowding ranging between about 10 and 50 passengers/train.

These results have been discussed with the panel of experts. It emerged that introducing seat reservation on suburban railway lines, although currently being tested on some regional lines, is not seen as effective and sustainable since it could induce a modal shift towards other modes (in particular car) in case of recurrently over-crowed trains and for those segments not familiar with smartphones (e.g. elderly). More radical solutions need to be implemented to reduce demand peaks such as changes in times of the schools, differentiated schedules in office and factories working hours, changes in the times of the cities (e.g. shopping, public services, medical cares,...). However, these may induce deep changes in households' habits and daily routines, thus requiring strong political and administrative actions, and, about this issue, the panel has shown little confidence in the ability of the public administration to manage these radical changes by implementing such measures, especially in an emergency situation (see Figure 13).

Among others, the absence of a systemic vision and bureaucracy are identified as the main criticalities. Among others, the absence of a systemic vision and the bureaucratic procedures for accessing public funds are identified as the main criticalities. Furthermore, the majority of respondents see the absence of full collaboration between Pubic administrations at local, regional and national levels as a barrier.

In the ex-urban case, the simulation has shown that keeping interpersonal distancing on board HSR train means halving capacity and even with demand levels of $50 \%$ or $40 \%$ of those PRE-Covid, there would be quite a significative number of unsatisfied demand due to seat unavailability in the peak periods. This would imply a significant loss in terms of revenues for the railway undertakings, which could only be compensated using public funds. In this respect, a general consensus about the importance of sustaining public transportation has been observed within the panel, due to the strategic importance of rail sector and due to the fact that an unmanaged increase of journeys by cars could lead to high level of road congestion, with severe consequences on the environment (landscape and air quality) and on people's quality of life. 


\section{Declarations}

\section{Availability of data and material}

Most datasets used and/or analyzed during the current study are available from the corresponding author on reasonable request. Restrictions apply to the availability of data that was used under license for the current study, and so are not publicly available. This data is however available from the authors with permission of the Trenitalia e Trenord companies.

\section{Funding}

Not Applicable

\section{Acknowledgements}

Not applicable

\section{Competing interests}

The authors declare that they have no competing interests.

\section{References}

Coppola, P. \& De Fabiis, F. (2020). Evolution of mobility sector during and beyond Covid-19: viewpoint of industries, consultancies and public transport companies. Tema. Journal of Land Use, Mobility and Environment, pp. 81-90. http://dx.doi.org/10.6092/1970-9870/6900

Cascetta E., Coppola P., Velardi V. (2013) High-Speed Rail Demand: Before-and-After Evidence from the Italian Market. disP-The Planning Review, Vol. 49:2, pp. 51-59.

https://doi.org/10.1080/02513625.2013.826539

Cascetta, E., Coppola, P. (2012) An elastic demand schedule-based multimodal assignment model for the simulation of high speed rail (HSR) systems. EURO Journal on Transportation and Logistics, Vol. 1, pp. 3-27. https://doi.org/10.1007/s13676-012-0002-0

Isfort (2020) "L'impatto del lockdown sui comportamenti di mobilità degli italiani" Research Report, 15 Maggio 2020 (www.isfort.it)

Ispos (2020) "Impact of Coronavirus to new car purchase in China" Research Report, 12 March 2020 (www.ipsos.com)

\section{Figures}



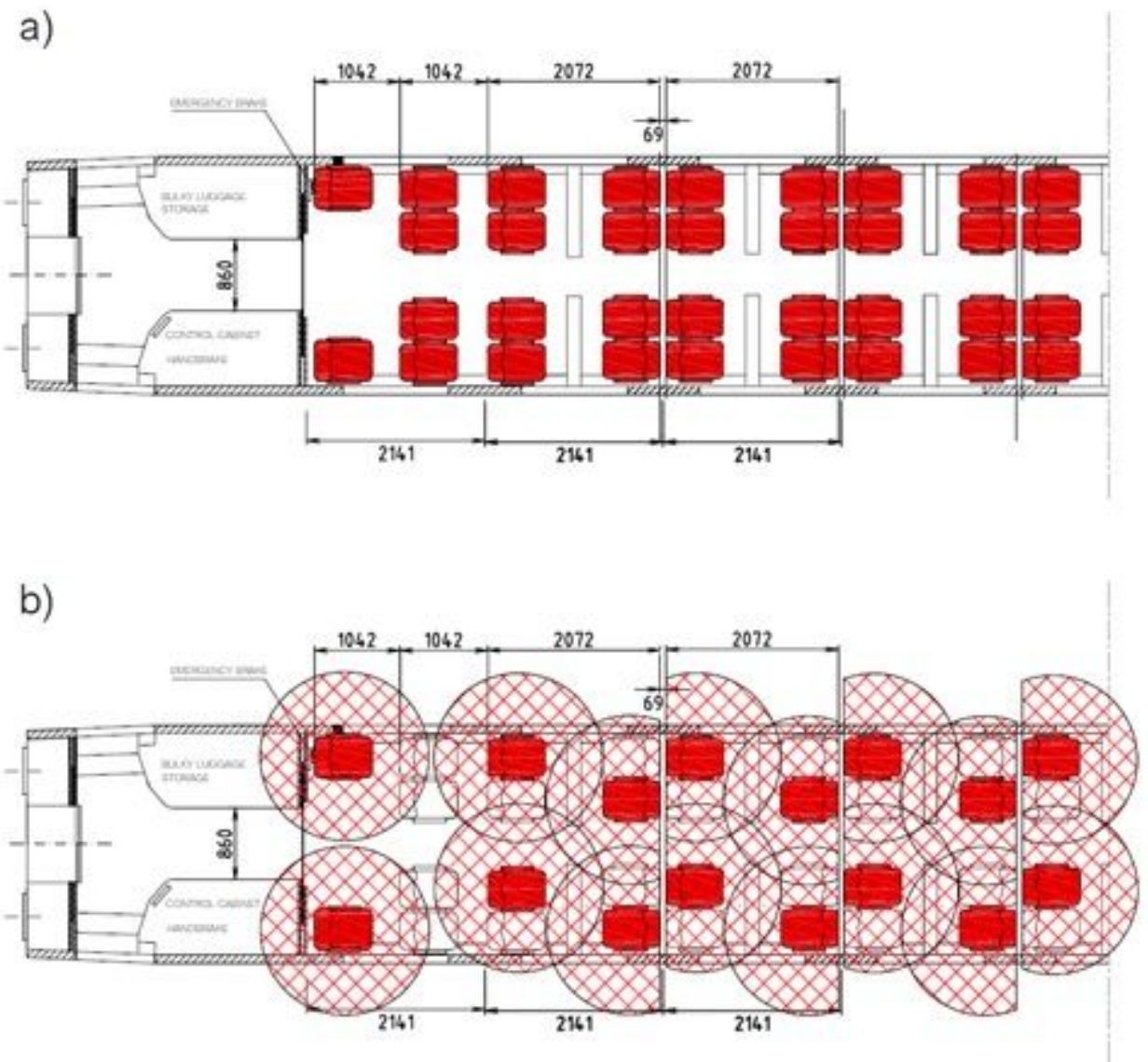

Figure 1

Example of available seats on an High-speed train ETR 500 Standard coach with full occupancy (a) and with one-meter interpersonal distancing (b): in red the seats available.

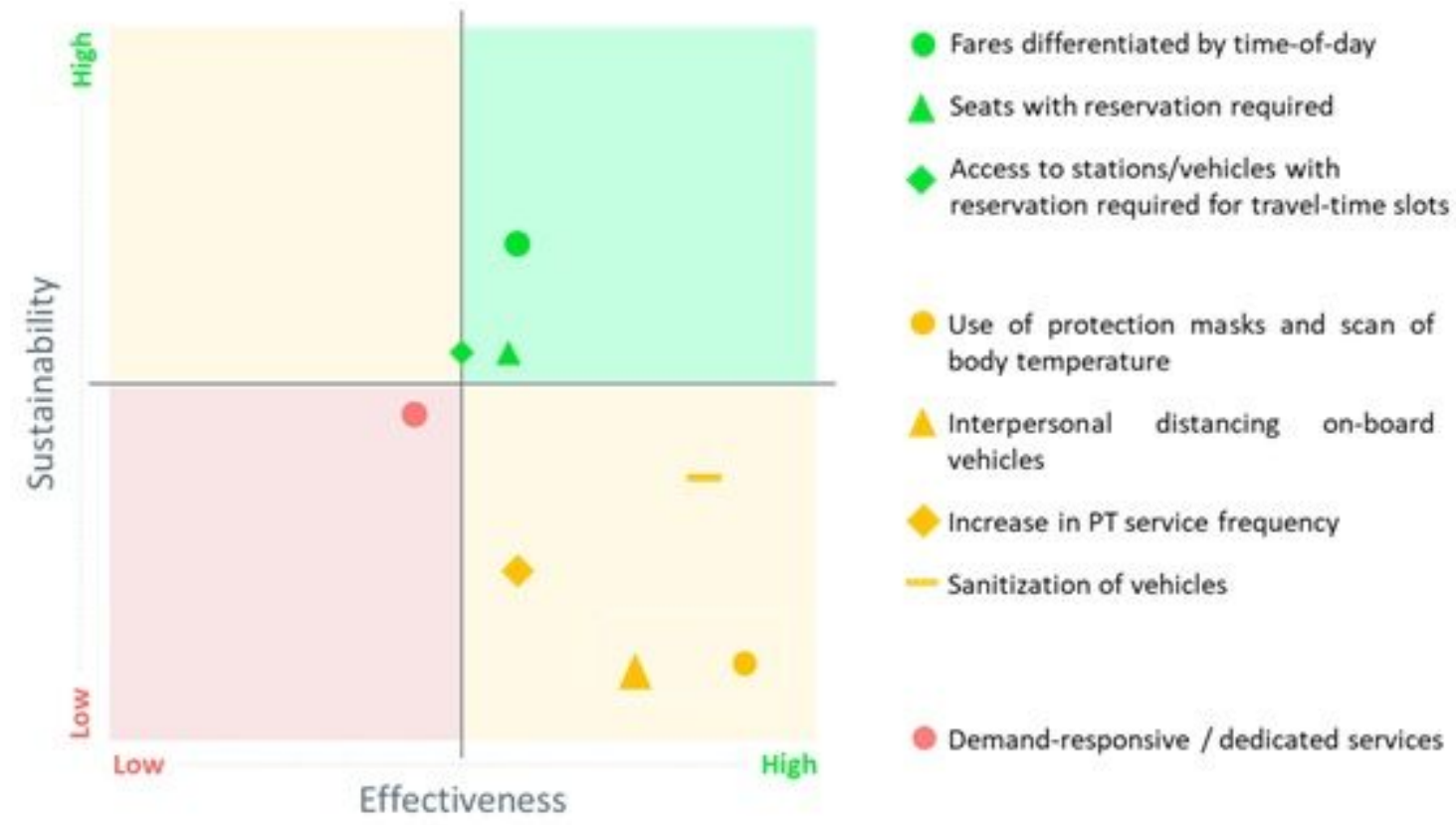

Figure 2 
Domains of effectiveness and sustainability of the proposed measures to cope with the COVID-19 health emergency (Coppola and De Fabiis, 2020)

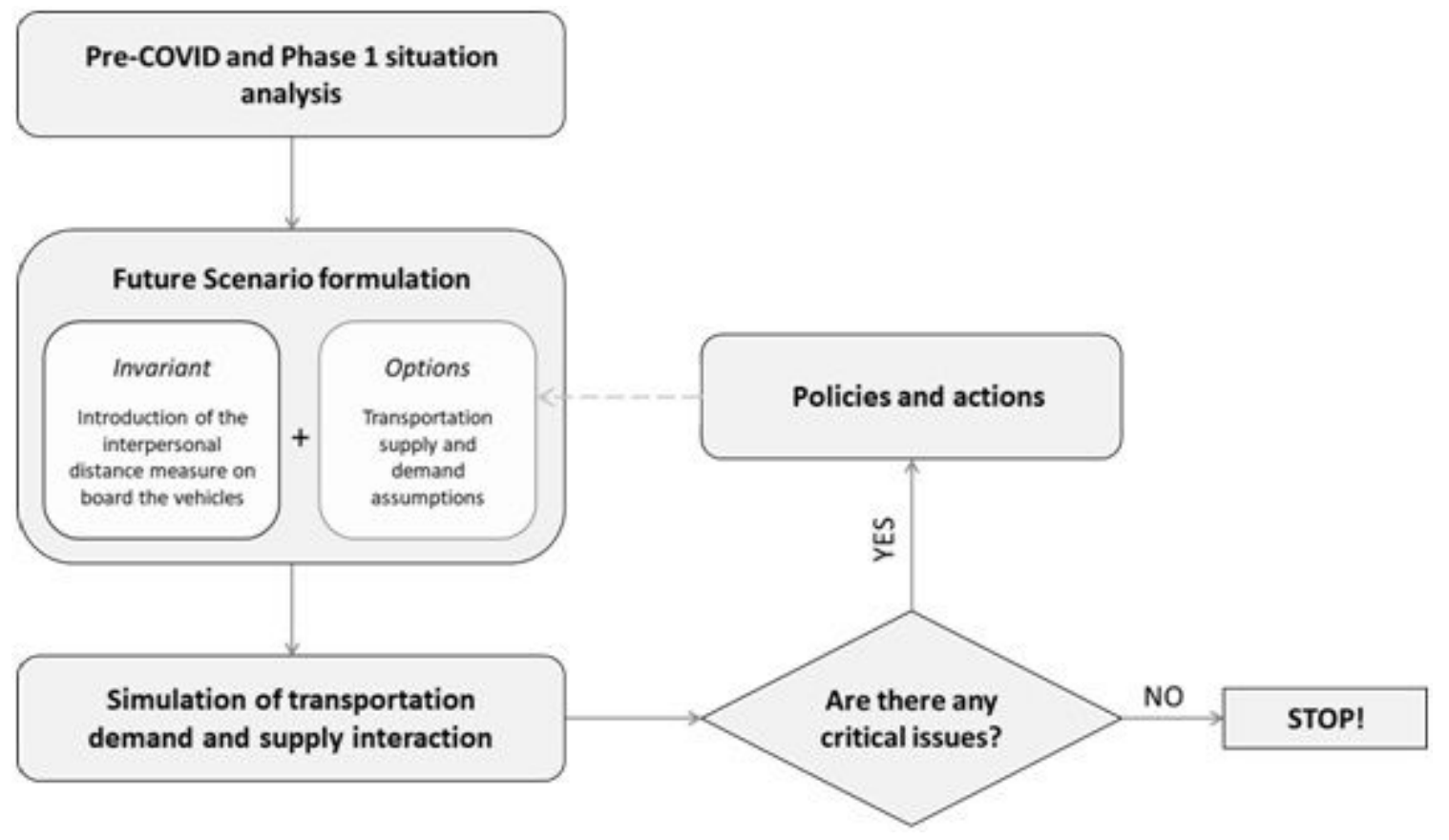

Figure 3

Schematic representation of the proposed methodology to identify PT system critical issues

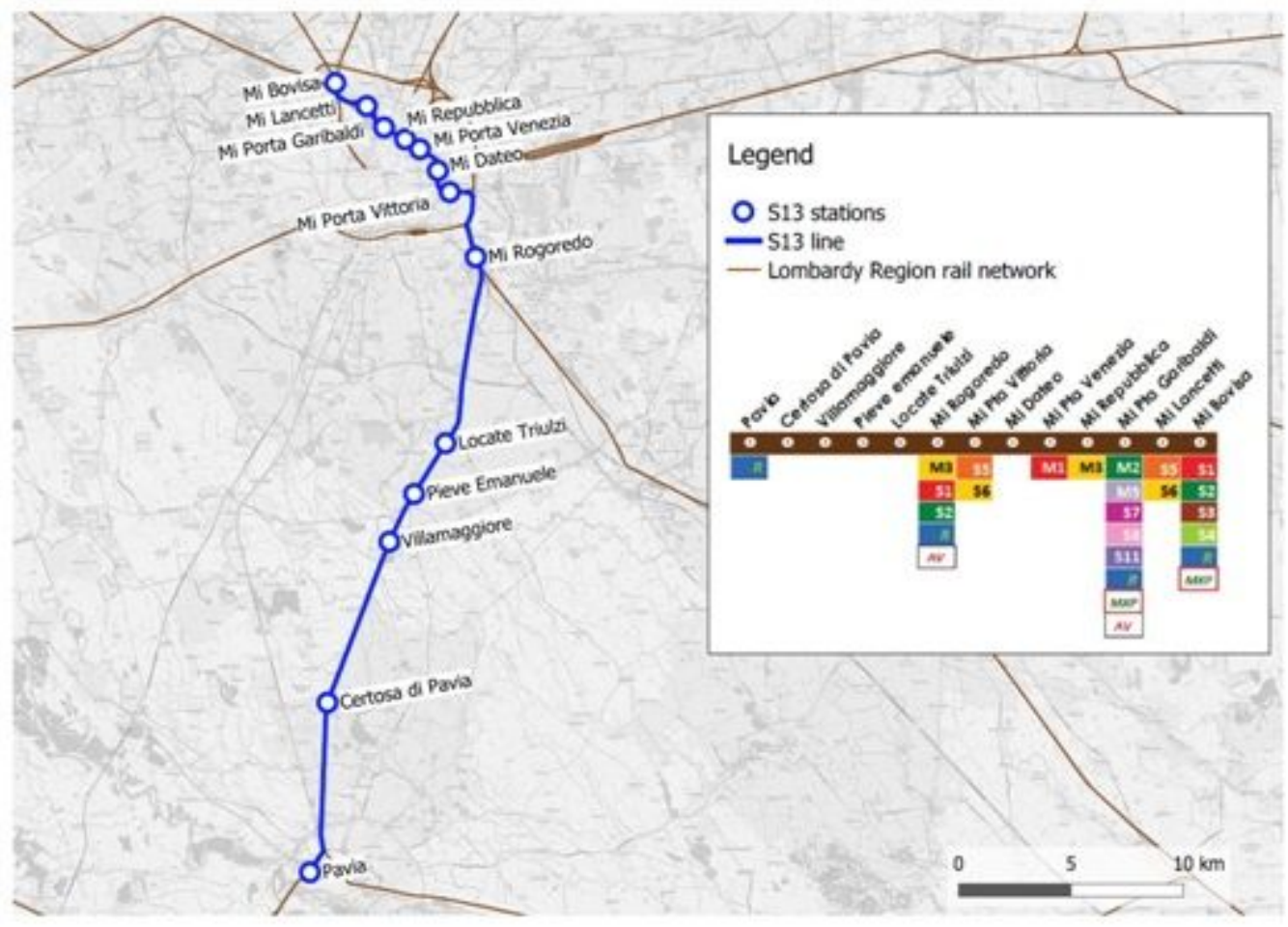

\section{Figure 4}

The $\mathrm{S} 13$ sub-urban railway line and interconnections with other metro $(\mathrm{M})$ and suburban $(\mathrm{S})$ lines 

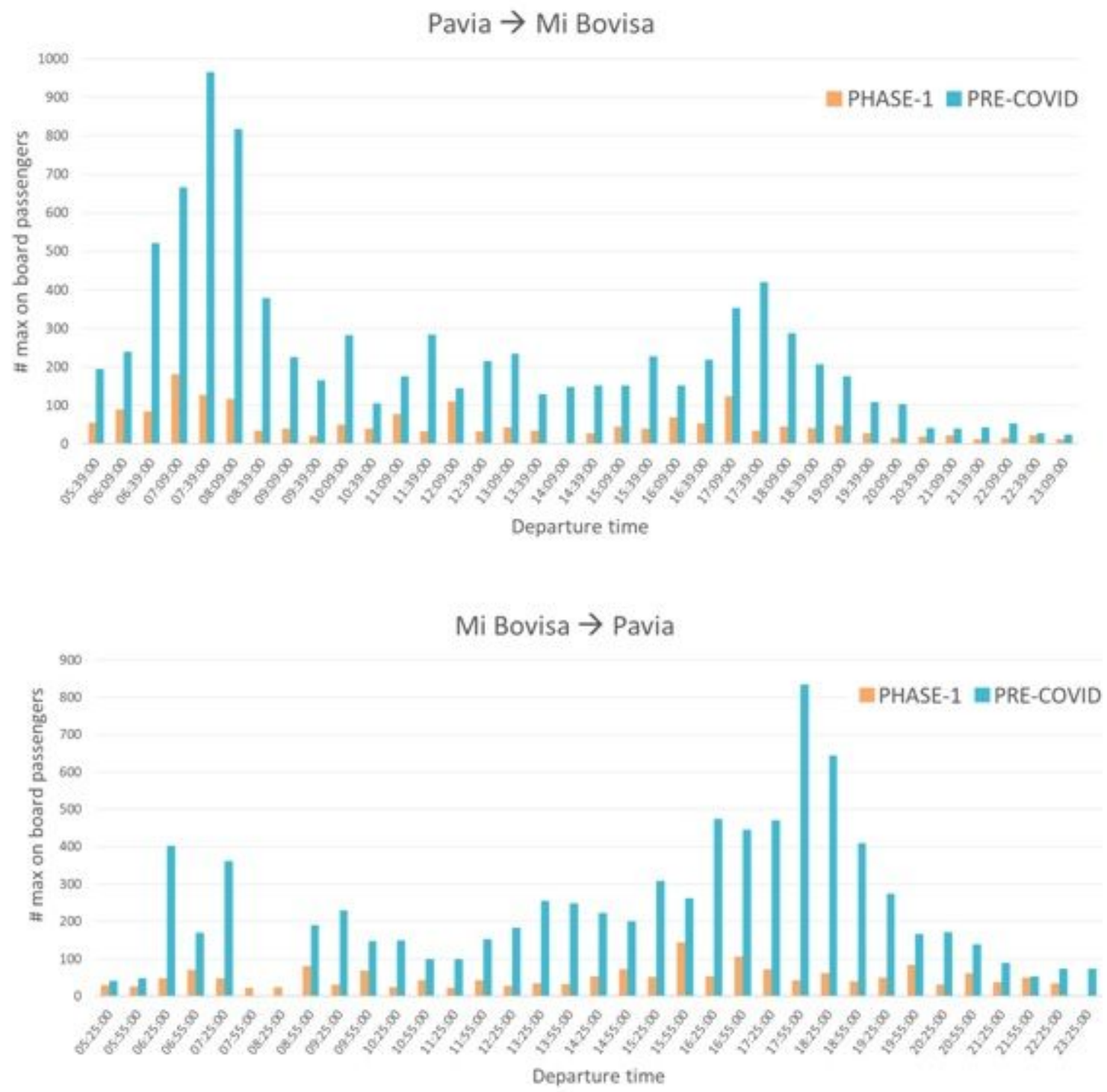

\section{Figure 5}

Maximum number of on-board passengers for each S13 run during a working day (Pavia - Mi Bovisa and Mi Bovisa - Pavia directions) 


\begin{tabular}{cc}
\hline$\#$ & Stotion \\
\hline 1 & Mi Bovisa \\
2 & Mi Lancetti \\
3 & Mi Porta Garibaldi \\
4 & Mi Repubblica \\
5 & Mi Porta Venezia \\
6 & Mi Dateo \\
7 & Mi Porta Vittoria \\
8 & Mi Rogoredo \\
9 & Locate Triulzi \\
10 & Pieve Emanuele \\
11 & Villamaggiore \\
12 & Certosa di Pavia \\
13 & Pavia \\
\hline
\end{tabular}
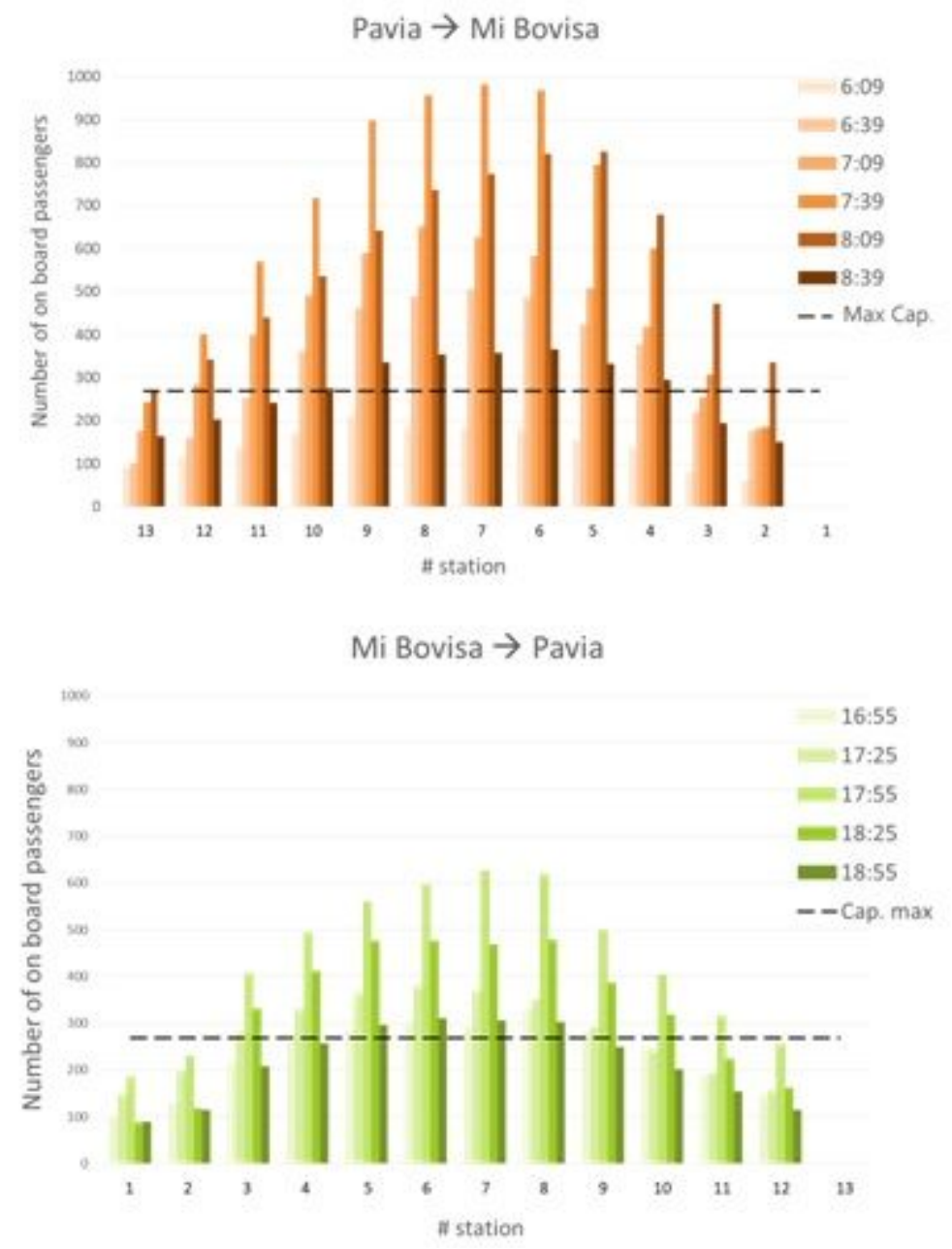

\section{Figure 6}

Flow diagram of passengers on-board the train of line S13 during the morning peak in the Pavia-Bovisa direction and in the evening peak in the Bovisa-Pavia direction ("PRE-COVID").

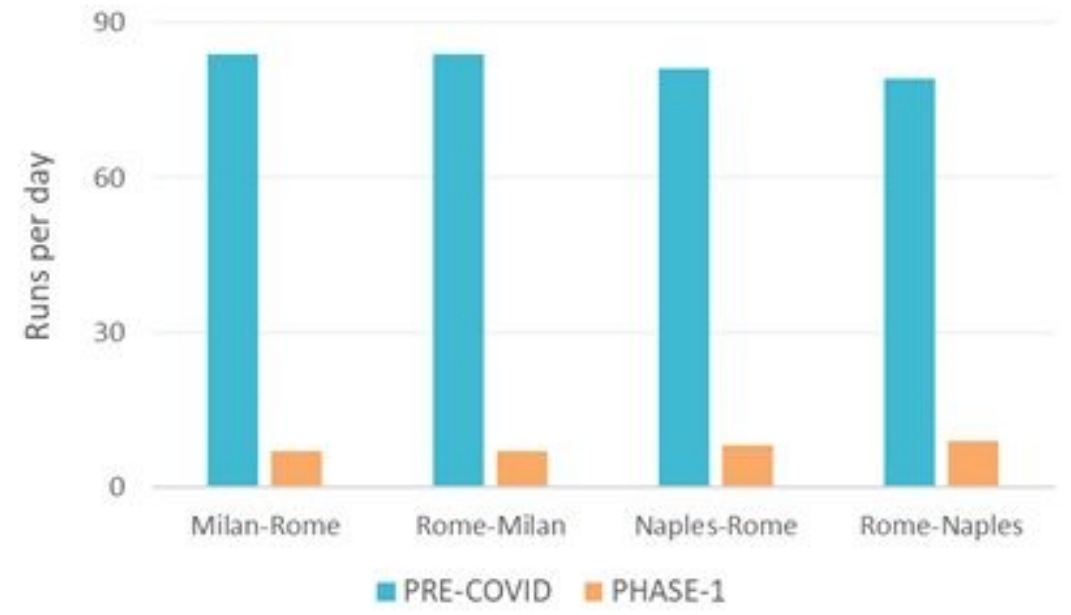

Figure 7 
Comparison of runs per day between the PRE-COVID and the PHASE-1 scenario for the different origindestination pairs under analysis

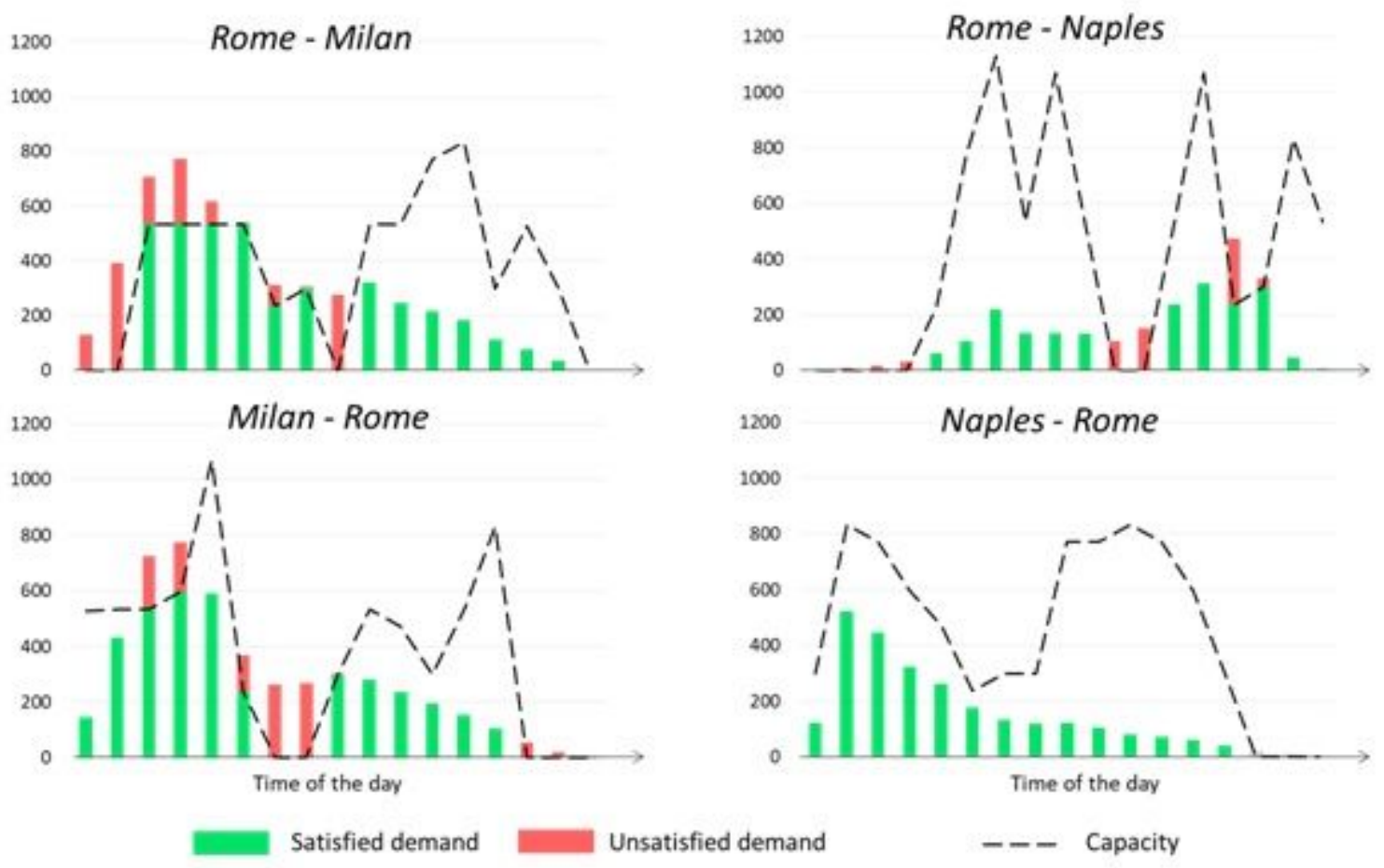

\section{Figure 8}

Estimated demand flows by hour-of -day in "SCENARIO 40\%"

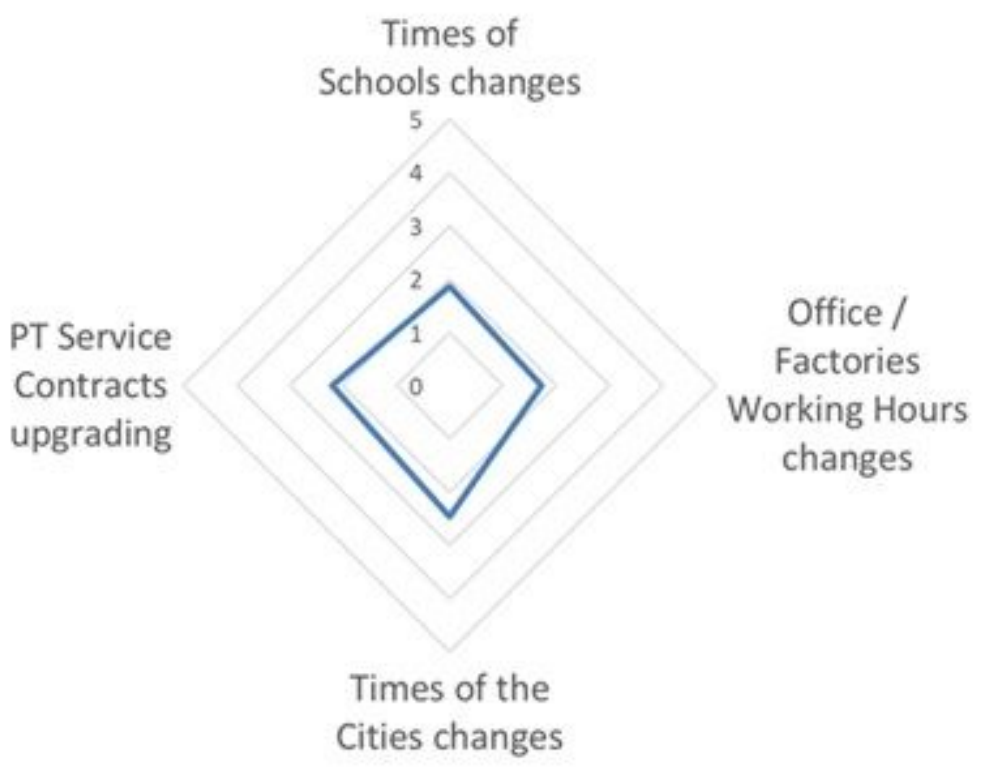

Figure 9 
Public administration capacity to implement strong policies to spread demand peaks and to upgrade PT service contracts in a Likert scale from $1=$ very low to $5=$ very high (Coppola and De Fabiis, 2020) 